\title{
Nubel, père de Sammac*, Firmus*, Gildon*, etc.
}

\section{Jean-Pierre Laporte}

\section{OpenEdition}

Journals

Édition électronique

URL : https://journals.openedition.org/encyclopedieberbere/2763

DOI : $10.4000 /$ encyclopedieberbere. 2763

ISSN : 2262-7197

\section{Éditeur}

Peeters Publishers

\section{Édition imprimée}

Date de publication : 5 octobre 2012

Pagination : 5626-5630

ISBN : 978-90-429-2718-6

ISSN : $1015-7344$

\section{Référence électronique}

Jean-Pierre Laporte, " Nubel, père de Sammac*, Firmus*, Gildon*, etc. », Encyclopédie berbère [En ligne], 34 | 2012, document N72, mis en ligne le 15 décembre 2020, consulté le 17 février 2022. URL : http:// journals.openedition.org/encyclopedieberbere/2763; DOI : https://doi.org/10.4000/ encyclopedieberbere. 2763

Ce document a été généré automatiquement le 17 février 2022.

(c) Tous droits réservés 


\title{
Nubel, père de Sammac*, Firmus*, Gildon*, etc.
}

\author{
Jean-Pierre Laporte
}

1 Des recherches récentes (Laporte, 2004 et à paraître) sur Nubel et sa famille ont écarté $\mathrm{du}$ dossier divers documents dont une inscription de Rusguniae (qui concerne un homonyme partiel et plus tardif), la dédicace dite du « col des Beni Aïcha » (sur laquelle le nom de Nubel n'était lu que par une restitution fort contestable), et enfin le mausolée de Blad Guitoun (plus tardif). Ceci a permis d'éliminer une bonne partie des hypothèses, notamment généalogiques, qui en découlaient. En revanche, le texte de la dédicace du praedium/praesidium de Sammac à M'lakou* permet de préciser la situation et la nature du pouvoir de Nubel. Des homonymes sont attestés à plusieurs reprises sur des inscriptions libyques et puniques, ainsi sous la forme $n b l$ sur deux stèles puniques de Constantine, mais aussi sur des inscriptions libyques (Chabot: RIL, 554, $572:$ NBLH), et enfin à l'époque romaine sur l'épitaphe de Félix, fils de Nibil, princeps des Suburbures (Lancel 1955). Le nom doit être libyque : la racine NBL est phonologiquement tout à fait possible en berbère, sans que son étude fournisse pour l'instant un sens précis ${ }^{1}$.

2 Selon Ammien Marcellin (XXIX, V, 44), Nubel était originaire de la tribu des Jubaleni. Le territoire de cette tribu était couvert de montagnes entre lesquelles s'ouvraient des gorges tortueuses, qui remplirent d'appréhension Théodose l'Ancien. St. Gsell, qui a noté que cette tribu devait être assez proche d'Auzia, a été tenté de la placer soit aux Portes de Fer, à $60 \mathrm{~km}$ à l'ouest d'Auzia), soit aux gorges de Lakhdaria, ex-Palestro (à $50 \mathrm{~km}$ au nord-ouest de la même ville) (S. Gsell 1903, p. 26 = 1981, p. 117). Les fondements logiques de la seconde proposition paraissent d'autant plus faibles que la dédicace du col des Beni Aïcha, qui en était l'un des arguments majeurs, est maintenant écartée du dossier. Dans la première solution, au contraire, le territoire des Jubaleni était très proche de l'emplacement du futur praedium/praesidium de Sammac à M'lakou.

Nubel, qui vivait vers le milieu du IV siècle, mourut à une date inconnue, en tout cas antérieure au déclenchement de la guerre de Firmus, dont les difficultés de sa succession furent la cause principale. Ammien souligne son importance politique (XXIX, V, 2) : Nubel, velut regulus per nationes Mauricas potentissimus, « le roi le puissant 
parmi les nations maures ». Cette désignation, un peu énigmatique et souvent sousestimée, est à notre sens pleinement expliquée par la dédicace du praedium/praesidium de son fils Sammac à M'lakou. Ce dernier l'avait construit comme une ville : fundus Petrensis, in modum urbis (Ammien Marcellin (XXIX, V, 13). L'identification est certaine. Pas moins de trois indices concordent : le nom du fundus, le nom de son propriétaire, et enfin la proximité de Tubusuctu. Sur la dédicace, l'édifice est désigné par les deux mots de praesidium, qui comporte une connotation militaire et de praedium qui désigne un domaine agricole, ce qui montre sa double nature. Premières et dernières lettres des huit lignes sont séparées du reste du texte par une hedera (ici : *), ce qui met bien en évidence le double acrostiche Praedium Sammacis. La virtuosité du lapicide a été à la hauteur de celle de l'auteur de ce véritable tour de force textuel.

\begin{tabular}{|c|c|c|}
\hline$P$ & * raesidium aeternae firmat prudentia paci* & $S$ \\
\hline$R$ & * em quoque romana fida tutat undique dextr* & A \\
\hline$A$ & * mni praepositum firmans munimine monte ${ }^{*}$ & $M$ \\
\hline E & * cuius nomen vocitavit nomine Petra* & $M$ \\
\hline$D$ & * enique finitimae gentes deponere bell* & $A$ \\
\hline I & *n tua concurrunt cupiente foedera, Samma* & c \\
\hline $\mathrm{V}$ & *t virtus comitata fidem concordet in omn* & $I$ \\
\hline M & *unere, Romaleis semper sociata triumphi* & $s$ \\
\hline
\end{tabular}

En voici une traduction due à D. Lengrand (1994) :

«La prévoyance d'une paix éternelle renforce cette place forte.

Celle-ci protège aussi de tout côté l'État romain par une aide loyale.

Préposée au fleuve, la forteresse renforce par un rempart le mont,

Et du nom de celui-ci, elle a pour nom Petra.

En conséquence, les peuples voisins désireux de cesser la guerre

Accourent en recherchant (la paix) dans tes traités, Sammac,

Afin que la bravoure, compagne de la fidélité, soit d'un seul cœur, toujours associée aux

triomphes des Romuléens [les Romains, nommés ici comme descendants de Romulus]. 


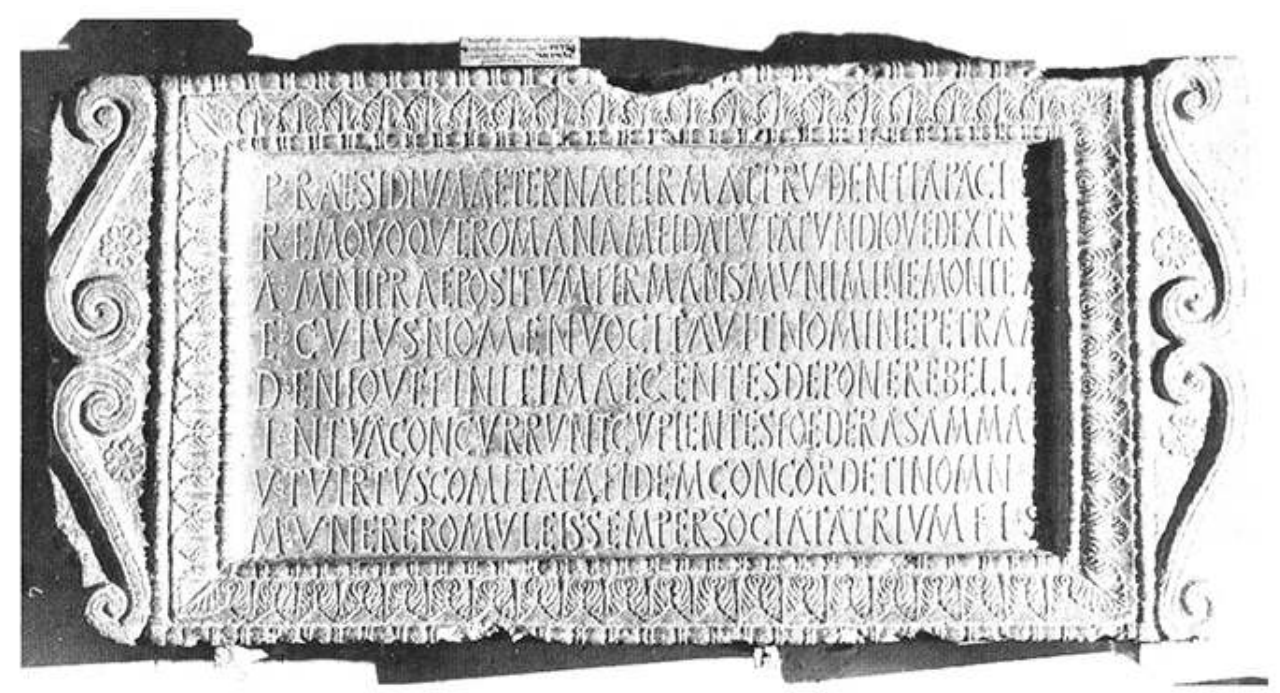

Fig. 1. M'LAKOU. LA DÉdICACE dU FUNDUS PETRENSIS, PRAEDIUM/PRAESIDIUM DE SAMMAC (fils DE NUBEL). AU MUSÉE D'ALgER.

Cliché Laporte, 1970.

5 La mission romaine de Sammac est bien définie : ramener les peuples de la région dans la paix romaine et les y maintenir par des traités. Cette fonction était un rouage essentiel pour le contrôle des parties tribales, c'est-à-dire les moins romanisées mais aussi les plus étendues, de la Maurétanie Césarienne. À notre sens, Nubel, qui avait déjà ce pouvoir, occupait donc une place très élevée dans l'administration romaine de la province de Maurétanie césarienne.

6 Nous connaissons nommément sept descendants de Nubel en première génération :

\begin{tabular}{|l|l|l|l|l|l|}
\hline Enfants & Ammien, XXIX, V & Origine du nom & Confession & Choix lors de la révolte & PLRE \\
\hline Sammac & 2 & Libyque & $?$ & (assassiné) & I, 2, p. 801. \\
\hline Firmus & Passim & Latin & Donatiste & Révolté & I, $1, \mathrm{p} .339$ \\
\hline Gildon & $6 ; 21 ; 24$ & Libyque & Donatiste & Côté romain & I, 1 p. 395. \\
\hline Mazucan & $40 ; 41$ & Libyque & $?$ & Révolté & I, 2, p. 591. \\
\hline Mascezel & $11 ; 14$ & Libyque & Catholique & Révolté, puis réconcilié & I, 2, p. 566 \\
\hline Dius & 11 & Latin $?$ & $?$ & Révolté & I, 1, p. 262 \\
\hline Cyria & 28 & Grécisant $?$ & $?$ & Révolté & I, 1, p. 237 \\
\hline
\end{tabular}

Les enfants de Nubel lors de la guerre de Firmus.

7 Lors de la révolte de Firmus contre Rome, les enfants de Nubel, quelle que soit l'origine de leur nom personnel, se situèrent dans l'un ou l'autre camp, c'est-à-dire qu'ils obéirent à des choix personnels. Manifestement, la famille était plutôt donatiste*, même si Mascezel* était catholique (rappelons que les différences entre les deux confessions étaient très minces dans les faits). 
On tire en général du texte d'Ammien Marcellin (XXIX, V, 2) une généalogie simple dans laquelle tous les enfants de Nubel sont souvent mis sur le même plan. Une petite incise du texte longtemps restée inaperçue suggère le contraire : "Nubel, en quittant la vie, laissa des fils légitimes et des fils nés de concubines ». Bien que probablement chrétien, si l'on en juge par la religion de ses enfants, Nubel était polygame, vieille tradition libyque que n'avait pu éliminer la christianisation de la famille. Ses enfants, issus d'une épouse légitime et de concubines, étaient de ce fait de statut différent. Si Ammien cite ce détail dans la première phrase de son récit, c'est parce qu'il était important pour la suite de son exposé : le sort différent réservé dans la succession de Nubel à ses enfants " provoqua discordes et guerres » (Ammien, ibidem).

Qui de Sammac ou de Firmus était l'héritier légitime? Ammien ne le précise pas. Il n'y a que deux solutions. On considère en général que Firmus était l'héritier légitime de Nubel. Mais on verrait mal dans ce cas qu'il n'ait pas hérité directement du pouvoir de son père et ait eu besoin pour ce faire d'assassiner son frère illégitime. Cette solution a été explorée en détail par D. Lengrand (1994) qui en est arrivé de manière tout à fait logique à une solution fort peu vraisemblable selon laquelle Sammac se serait mis dans la clientèle de Romanus pour compenser sa bâtardise, et selon laquelle il se serait vanté dans la dédicace de son fundus d'arrêter des guerres tribales qu'il avait lui-même suscitées au préalable (sic).

En revanche, tout s'explique bien plus naturellement si Sammac était l'héritier légitime de Nubel. Il est normal que l'on retrouve alors entre ses mains l'influence et le rôle hérités de son père et bien expliqués sur la dédicace de son praedium/praesidium. On comprend bien que son ambitieux demi-frère Firmus l'ait assassiné pour récupérer position sociale et pouvoir. De même, on comprend bien la réaction vive du comte Romanus devant l'assassinat de l'un de ses principaux auxiliaires, rouage essentiel du contrôle romain des tribus de Maurétanie césarienne.

11 Il ne s'agissait pas d'une simple querelle de succession au sein d'une tribu maure, mais bien d'un conflit qui touchait directement l'administration romaine de la province sur un point particulièrement sensible: l'encadrement des tribus, à une époque où l'ascension sociale des chefs autochtones débouchait sur la mise en place progressive d'une sorte de féodalité. Selon un mot de Pierre Salama (1954, p. 229) : « à la veille de la guerre de Firmus qui devait ravager la province entière, la puissance romaine, sous le couvert de son organisation administrative, se trouvait à la merci des seigneurs locaux ».

12 Bien plus qu'un seigneur local «habituel», Nubel faisait partie de la haute administration provinciale romaine, par (et pour) laquelle il était chargé de l'encadrement des tribus, compte tenu de son origine autochtone. Son fils légitime Sammac hérita de sa fonction et de sa position. Son fils illégitime Firmus tua son demifrère pour récupérer ce pouvoir, déclenchant ainsi la révolte qui allait porter son nom. Un autre de ses fils, Gildon*, allait faire une carrière brillante à Rome, devenir comte d'Afrique, et entrer dans l'entourage immédiat de l'empereur en mariant sa fille Salvina à l'un de ses neveux, bel exemple de l'ascension de grands officiers d'origine barbare dans l'Empire de la fin du IV ${ }^{\mathrm{e}}$ siècle. 


\section{BIBLIOGRAPHIE}

PLRE : Prosopography of the Later Roman Empire, Ed. Arnold Hugh Martin Jones, John Martindale \& John Morris, Cambridge University Press, t. I, 1971.

RIL : J.-B. Chabot, Recueil des inscriptions libyques, Paris, Imprimerie nationale, 1940/41.

AMmIEN MARCELLIN, Histoires, 1. XXIX-XXXI, éd. et trad. Guy Sabbah, Paris, Belles Lettres 1999, 370 p. CAMPS G., 1997 - s.v. Firmus, EB, XIX, p. 2845-2855.

CSELL S., 1903 - « Observations géographiques sur la révolte de Firmus », RSAC, 36, p. 21-46 = Id., “Scripta varia”, 1981, p. 113-137.

GSELL S., 1901 - « Note sur une inscription d'Ighzer Amokrane », CRAI, p. 170-172.

KHERBOUCHE F., 2010 - s.v. « M’lakou », EB, XXXII, p. 5032-5033.

LANCEL S., 1955 - «Suburbures et Nicives : une inscription de Tigisis », Libyca, Archéologie/épigraphie, III, 1955, p. 294-295.

LAPORTE J.-P., 2004 - « Les armées romaines et la révolte de Firmus (370-373), in Congrès L'Armée romaine de Dioclétien à Valentinien Ier, 2002 (2004), Lyon, p. 269-288.

LAPORTE J.-P., à paraître - « Nubel, Sammac, Firmus et les autres », Africa romana, t. 19, 2010, à paraître (2013).

LENGRAND D., 1994 - «L'inscription de Petra et la révolte de Firmus », BCTH, n. s., B, 23, 1994, p. $159-170$.

SALAMA P., 1954 - « Inscription maurétanienne de 346 [trouvée à la Kherba des Aouissat] », Libyca a./é., II, 1,1954, p. 205-229),

\section{NOTES}

1. La racine NBL est attestée au moins en touareg; cf. K.-G. Prasse et al., Dictionnaire touaregfrançais, Copenhague, Museum Tusculamnum Press, 2003, p. 583-584: ənbəl =1. « frapper à la bouche, à la mâchoire "; 2. "placer à côté de, juxtaposer ». Attention, ne pas confondre avec enḅəl (touareg) « enterrer », qui est issu de əmḍl. S.C.

\section{INDEX}

Mots-clés : Antiquité, Kabylie, Onomastique 